\title{
Quantifying numerical dispersion in non-orthogonal FDTD meshes
}

\author{
R. Nilavalan, I.J. Craddock and C.J. Railton
}

\begin{abstract}
Numerical electromagnetic models such as FDTD are widely used for the design and analysis of structures, including antennas. Numerical dispersion is one of the main sources of error that degrade the accuracy of the results - for each structure of interest, the users of the model must attempt to generate a mesh that will avoid introducing high levels of dispersion. This is, however, especially difficult for non-orthogonal meshes since little information is available on the dispersion properties of the non-orthogonal FDTD algorithm on complex meshes. For the first time, the dispersion in realistic non-orthogonal FDTD models of microstrip structures is quantified directly through numerical simulations. A test structure is considered, discretised using a number of nonorthogonal mesh configurations, including single and multiple skew angles. A numerical analysis of reflections generated at the transition between two mesh regions with different skew angles is also presented. These results give a practical guide to mesh generation for users of the algorithm.
\end{abstract}

\section{Introduction}

Finite-difference time-domain (FDTD) methods, originally proposed by Yee [1], have proved efficient numerical algorithms for the design and analysis of broadband antenna structures. However, the main limitation of the classical FDTD method is its restriction to orthogonal grids-this restriction makes it very difficult to model curved surfaces and edges accurately (such as might be found in a conformal array, for example).

A number of FDTD-based methods have been proposed that are suitable for curved structures. These include the contour path FDTD (CPFDTD) [2], non-orthogonal FDTD [3], and discrete surface integral (DSI) [4] methods.

This contribution focuses on the non-orthogonal FDTD method. This method is based upon a discretisation of Maxwell's curl equations in local curvilinear co-ordinates on a structured mesh. When this mesh is non-orthogonal, however, additional errors (notably dispersion and spurious reflection) are introduced into the results-consideration of these errors for realistically complex non-orthogonal meshes has received little attention in the literature.

The aim of this contribution is to quantify these errors for realistic meshes in order to guide users of the algorithm in their choice of mesh resolution and geometry. In this paper, therefore, for the first time the dispersion in the NonOrthogonal FDTD method is determined directly through simulation of a microstrip line (the results are compared with theoretical predictions for plane wave propagation, in the simple cases where these exist [5-7]). A numerical analysis of spurious numerical reflections generated at the transition between two mesh regions with different skew angles is also presented.

(C) IEE, 2002

IEE Proceedings online no. 20010144

DOI: $10.1049 / \mathrm{p}$-map:20010144

Paper first received 9 th March 2001 and in revised form 10th December 2001

The authors are with the Centre for Communications Research, University of Bristol, Merchant Venturers Building, Woodland Road, Bristol BS8 IUB, UK

\section{Closed-form numerical dispersion relation}

In a numerical solution of Maxwell's equations, the number of mesh cells per wavelength generally affects the propagation velocity of waves in the structure of interest. This phenomenon is known as numerical dispersion and may be characterised by deriving a numerical dispersion relation (NDR) for the algorithm. The NDR for Yee's orthogonal FDTD algorithm was derived analytically by Taflove [8], and yields the minimum grid resolutions that will give reasonably accurate results (commonly ten cells per wavelength is considered sufficient in free space).

A closed-form NDR for the non-orthogonal FDTD algorithm is, however, considerably more difficult to derive, since the algorithm includes additional complexities such as interpolation/averaging [9]. The NDR for the non-orthogonal FDTD algorithm is therefore only available for plane-wave propagation in a uniformly skewed mesh with a single skew angle $\theta$ [5-7]. Considering a uniformly-skewed mesh as shown in Fig. 1, this NDR is:

$$
\begin{aligned}
& \frac{\sin ^{2}(0.5 \omega \Delta t)}{c^{2} \Delta t^{2}}=\frac{1}{\sin ^{2} \theta}\left\{\frac{1}{\Delta x} \sin ^{2}\left(\frac{k \cos \phi \Delta x}{2}\right)\right. \\
& +\frac{1}{\Delta z} \sin ^{2}\left(\frac{k \cos (\phi-\theta) \Delta z}{2}\right) \\
& \left.\frac{\cos \theta}{2 \Delta x \Delta z} \sin (k \cos \phi \Delta x) \sin (k \cos (\phi-\theta) \Delta z)\right\}
\end{aligned}
$$

where $\omega$ is the temporal frequency (in $\mathrm{rad} \mathrm{s}^{-1}$ ), $\phi$ is the wave propagation angle measured from the $x$-axis, $\Delta x$ and $\Delta z$ are the mesh dimensions (see Fig. 1), and $k$ is the spatial wave number.

For a given value of $\omega$ and $\phi$, the resulting wave number for the numerical solution can be found by numerically solving this implicit relationship for $k$. The non-physical dependance of $k$ on $\phi, \theta, \Delta x$ and $\Delta z$ is the numerical dispersion. For practical problems, however, the nonorthogonal meshes used are much more complex and cannot be characterised by a single, uniform skew angle-for these meshes a closed-form NDR is not available and the numerical dispersion must instead be 


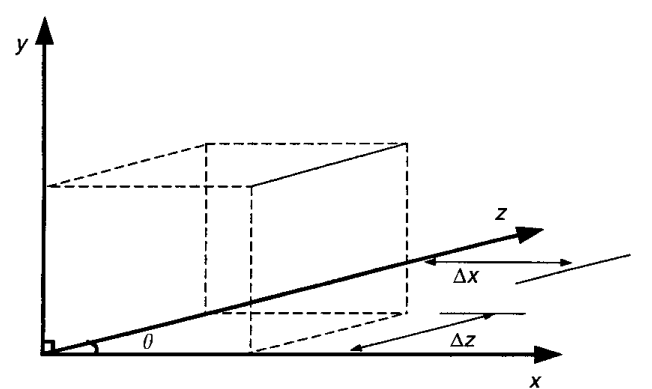

Fig. 1 Skewedmesh geometry

determined directly through numerical experiment, as described in Section 3.

\section{Non-orthogonal FDTD analysis}

Fig. 2 shows an air-spaced (non-dispersive) microstrip line used to analyse the numerical dispersion characteristics. The problem space was limited by employing absorbing boundaries and an electric wall to represent the ground plane. A Gaussian pulse of 300ps width was used to excite the problem and the electric fields were observed at four different probe locations. The microstrip line was made sufficiently long to avoid any influences from the absorbing boundary conditions. The problem space was discretised into $140 \times 25 \times 20$ unit cells with cell dimension $\Delta x=8.5 \mathrm{~mm}$ in the longitudinal direction.

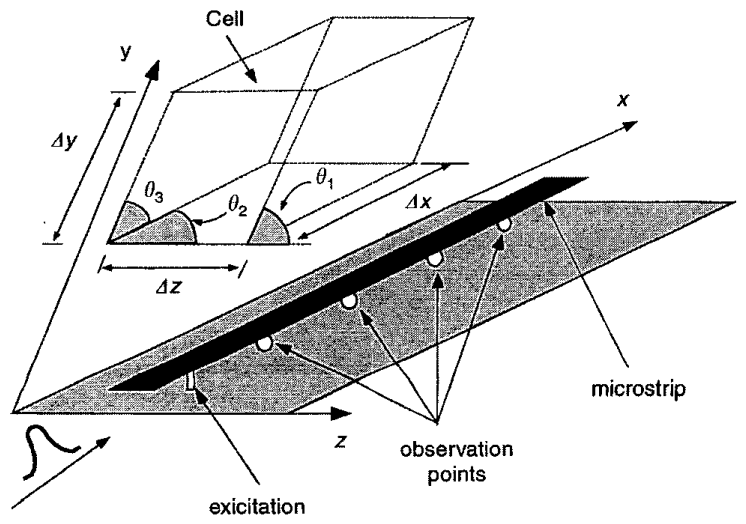

Fig. 2 Microstrip line and description of skewed cells

Once the FDTD temporal response has been obtained at four observation points located along the axis of propagation, the wave number $k$ was calculated using DFTs of the response [10]. An average value of $k$ was then calculated using all possible pairs of observation points, in order to improve accuracy. This procedure was repeated for three basic test cases (one, two and three angles of skew) and for each given test case the angles of skew were altered (Table 1).

Test case 1 consisted of a mesh with a single, uniform skew angle, in order that an initial comparison with the available closed-form NDR (1) could be accomplished. Fig. 3 shows the normalised wave number for case 1 against the mesh cell sizes (normalised with respect to the wavelength). The analytical estimations for this simple case, calculated using (1) (with $0^{\circ}$ propagation angle) are also presented in this figure.
Table 1: Test cases

\begin{tabular}{llll}
\hline & $\theta_{1}$ & $\theta_{2}$ & $\theta_{3}$ \\
\hline $\begin{array}{l}\text { Case } 1 \text { (skewed } \\
\text { with respect to }\end{array}$ & fixed: $90^{\circ}$ & $\begin{array}{l}\text { varied: } \\
\theta=90^{\circ}, 70^{\circ},\end{array}$ & fixed: $90^{\circ}$ \\
$\begin{array}{l}5 \text { axis) } \\
55^{\circ}, 45^{\circ}\end{array}$ & \\
Case 2 (skewed & varied: & $\begin{array}{l}\text { varied: } \\
\theta=70^{\circ}, 60^{\circ},\end{array}$ & fixed: $90^{\circ}$ \\
with respect to & $\theta=70^{\circ}, 60^{\circ}$, & $50^{\circ}$ & \\
2 axes) & $50^{\circ}$ & varied & \\
Case 3 (skewed & varied: & $\theta=70^{\circ}, 60^{\circ}$, & $\theta=70^{\circ}$, \\
with respect to & $\theta=70^{\circ}, 60^{\circ}$, & $50^{\circ}$ & $60^{\circ}, 50^{\circ}$ \\
3 axes) & $50^{\circ}$ & & \\
\hline
\end{tabular}

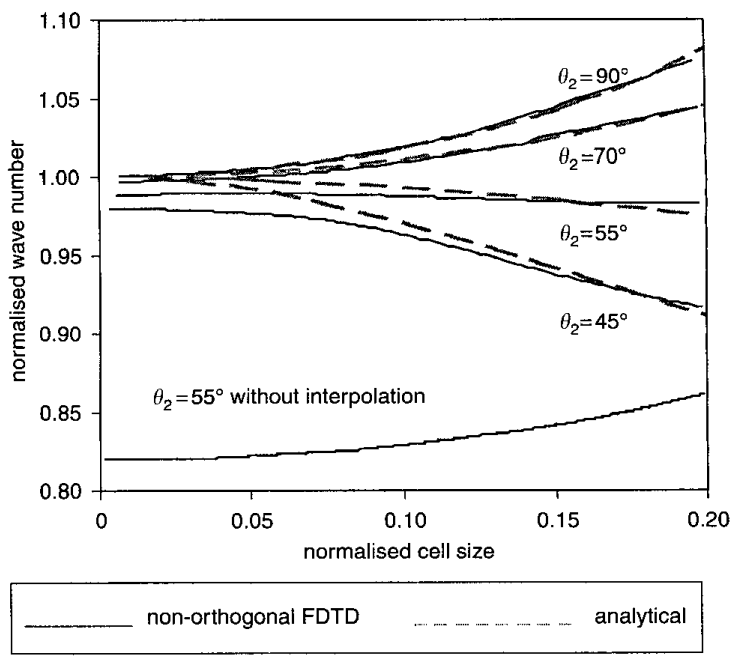

Fig. 3 Dispersion curves for varying $\theta_{2}$ with $\theta_{1}=\theta_{3}=90^{\circ}$ : case 1

Good agreement is seen between the analytical and the FDTD results for grid resolutions around ten cells per wavelength, although there is a persistent discrepancy for small skew angles at grid resolutions better than ten cells/ wavelength (this discrepancy is discussed in Section 5). It can be observed that the normalised wave number on moderately skewed meshes is closer to unity than it is for orthogonal meshes (for $0^{\circ}$ propagation), and then rapidly decreases with the skew angle.

The observed dispersive behaviour of the non-orthogonal mesh places restrictions on the usable angles that would yield reasonably accurate results. Taking a standard, Cartesian FDTD as a guide $\left(\theta_{2}=90^{\circ}\right.$ in case 1$)$, a grid resolution of better than ten cells/wavelength is usually recommended for accurate results-this guideline (as shown in Fig. 3) corresponds to approximately $2 \%$ dispersion error. If the same level of accuracy is required from the non-orthogonal method, Fig. 3 implies that the skew angle $\left(\theta_{2}\right)$ should be in the range $55^{\circ}$ to $90^{\circ}$ if a grid resolution of ten cells/wavelength is retained.

As described above, the non-orthogonal FDTD algorithm includes an interpolation/averaging procedure that transforms the available contravariant field components to covariant components in the correct position. It is interesting to note the effect of omitting this procedure - the resulting dispersion for $\theta_{2}=55^{\circ}$ is included in Fig. 3 and is particularly severe. This result shows the importance of the interpolation scheme in controlling dispersion in nonorthogonal meshes. 
Figs. 4 and 5 show the dispersion on meshes with multiple skew angles (cases 2 and 3), which is shown here for the first time using the non-orthogonal FDTD simulations. Dispersion becomes noticeably more pronounced as the grid becomes less orthogonal in all directions-as seen in Figs. 4 and 5, the normalised wave number remains less than 0.9 for angles smaller than $60^{\circ}$ for even very fine meshes.

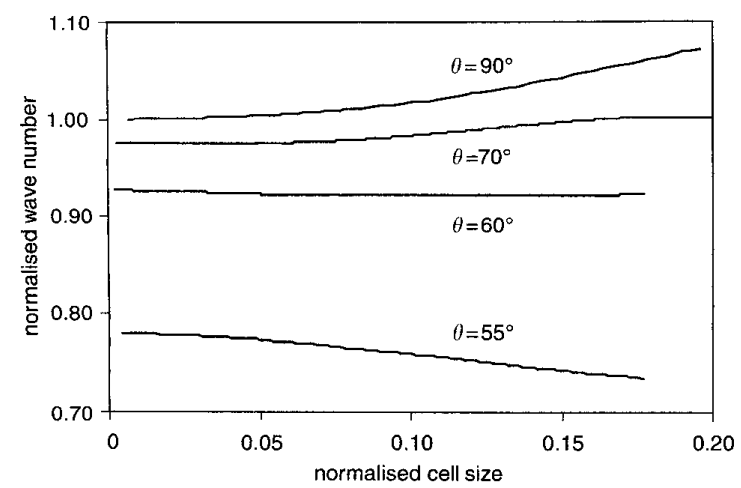

Fig. 4 Dispersion curves for varying $\theta\left(=\theta_{2}=\theta_{3}\right)$ with $\theta_{1}=90^{\circ}$. case 2

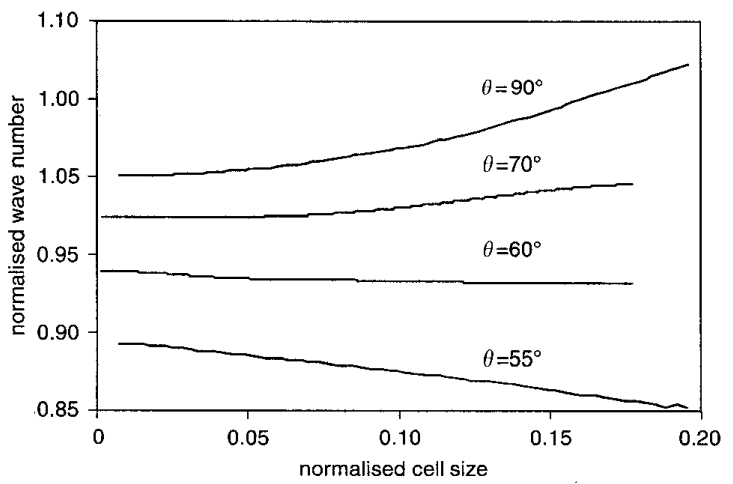

Fig. 5 Dispersion curves for varying $\theta\left(\theta_{l}=\theta_{2}=\theta_{3}\right)$ : case 3

It is interesting to note that the dispersive behaviour is worse for $\theta=50^{\circ}$ for the mesh with two skew angles (case 2, Fig. 4) than for the mesh with all three skew angles (case 3, Fig. 5); this behaviour may be due to the asymmetrical geometry of the cells. Apart from this behaviour, similar dispersion is seen for angles between $90^{\circ}$ and $60^{\circ}$.

It can be concluded that mesh skew angles smaller than $70^{\circ}$ result in significantly higher dispersion, making it difficult to employ them in non-orthogonal meshes with two and three skew angles. Considering propagation along one axis in a mesh with two or three skew angles, $\theta$ should be in the region between $70^{\circ}$ and $90^{\circ}$ for grid resolutions of ten cells/wavelength to give dispersion similar to the orthogonal FDTD method.

In practice, non-orthogonal FDTD meshes are likely to contain cells with a range of angles, rather than being uniformly skewed. It would be expected, however, that the dispersion data provided for the uniformly skewed case is a good guide to the behaviour of a mesh containing a variety of cell angles. This may be confirmed by considering a mesh gradually skewed from $90^{\circ}$ to a worst-case angle of $45^{\circ}$ half way along the microstrip line as shown in Fig. 6. In this case the dispersion is naturally a function of position on the line.
Fig. 7 shows dispersion curves calculated using observation points 2 and 3 and also the average dispersion that is observed along the microstrip line.

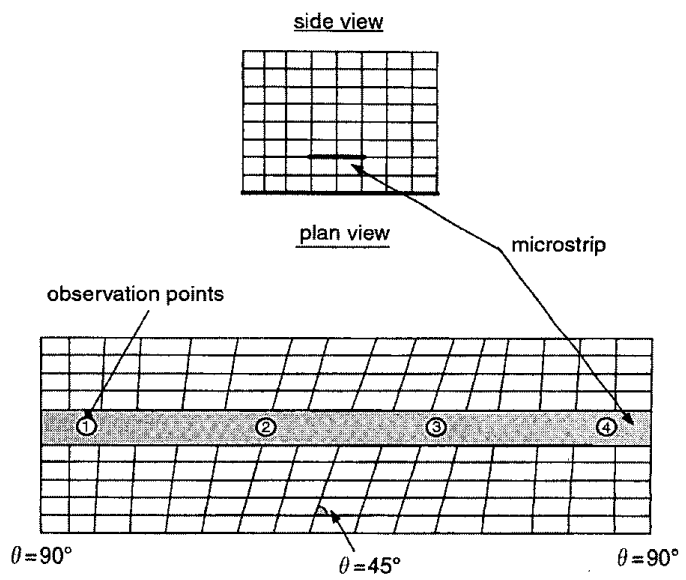

Fig. 6 Microstrip line with non-uniformly skewed mesh
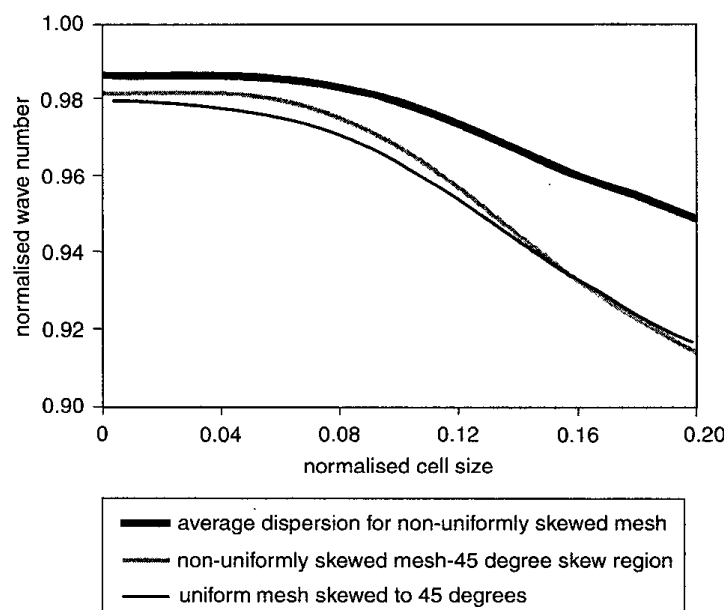

Fig. 7 Dispersion curves for propagation in non-uniformly skewed mesh

These results indicate that the local dispersive behaviour is dominated by the worst-case skew angle in each region and is similar to the dispersion from a uniformly skewed mesh (also included in Fig. 7). The average dispersion for the line is, however, as expected, less severe than the worstcase.

In a mesh containing a range of cell geometries, therefore, the studies of uniformly-skewed meshes presented herein are a good guide to local dispersion in each part of the mesh. The overall dispersion properties of such mesh will be bounded by (less severe than) the dispersion properties of the most distorted portion of the mesh.

\section{Reflections}

Since signals travel at different numerical velocities in different skew regions, reflections can be expected at the interface between these regions. These unwanted reflections are another potential source of error in non-orthogonal FDTD computations. Wave reflections associated with non-orthogonal meshes have been theoretically analysed by 
other authors [11], but their results ignored the interpolation/averaging step in the algorithm. In this Section, reflections from interfaces between different skew regions are analysed with a full non-orthogonal FDTD simulation, incorporating the interpolation step, which, as discussed in Section 3, is an important element in the algorithm.

These reflections were again estimated using a microstrip line with a skewed mesh that includes a sudden change in angle $\left(90^{\circ}\right.$ to an angle $\theta$ ). This sudden change avoids multiple reflections that can arise in a mesh with gradually changing angle. As shown in Fig. 8, this change in the angle requires different cell sizes at the interface and in the skewed

side view

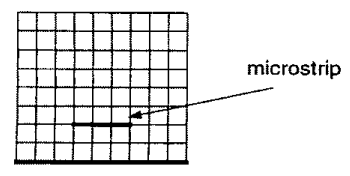

plan view

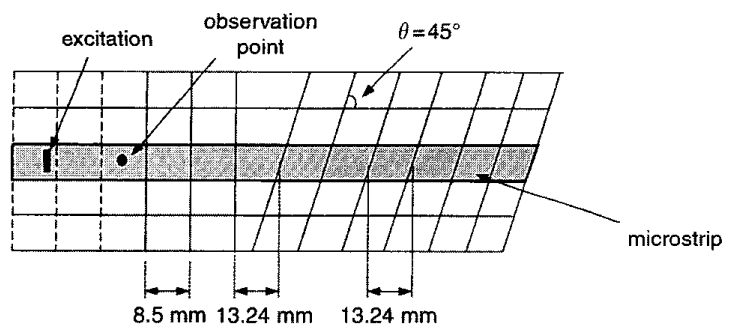

Fig. 8 Microstrip line for reflection estimation

region. Numerical investigations were carried out with both horizontally and vertically skewed meshes.

The source of reflection in this particular mesh is due to both the skew angles and to the different cell sizes inside the microstrip region. Reflections due to the change in cell sizes can be estimated using an orthogonal mesh with the same cell sizes. The reflection coefficients were calculated using Fourier transformation of the incident and reflected pulses at the observation location, and are shown in Fig. 9 (horizontal skew) and Fig. 10 (vertical skew) against the larger cell size ( $13.24 \mathrm{~mm}$ normalised by the wavelength). Calculations were performed for skew angles of $\theta=60^{\circ}$ and

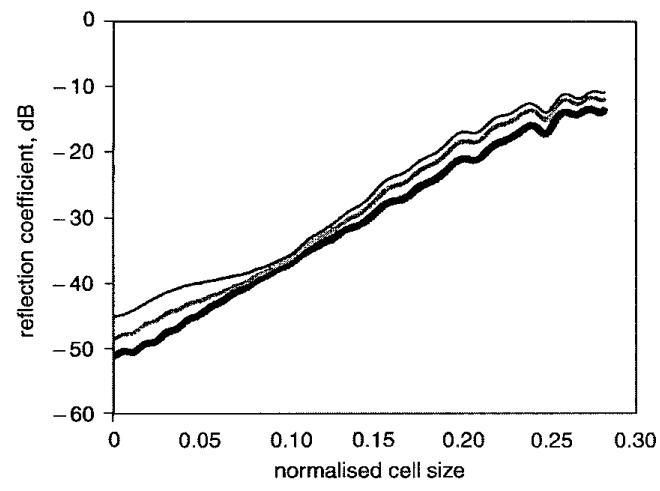

90 degrees -60 degrees $\quad 45$ degrees

Fig. 9 Reflections from skew regions with horizontally skewed grid

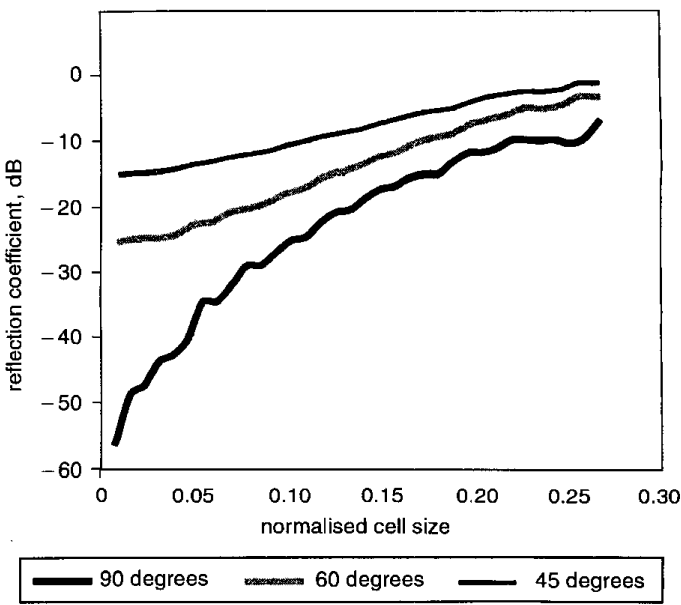

Fig. 10 Reflections from skew regions with vertically skewed grid

$45^{\circ}$, and the results were compared with an orthogonal mesh with the same cell sizes.

Fig. 9 shows the reflections from the horizontally skewed interface are less than $-35 \mathrm{~dB}$ for grid resolutions smaller than ten cells/wavelength for both orthogonal and nonorthogonal FDTD algorithms. Furthermore, it can be observed from Fig. 9 that the reflections from different skew regions increase with skew angles, in line with Fig. 3. The differences between the orthogonal mesh and the skew mesh have a maximum of $2 \mathrm{~dB}$ for the $60^{\circ}$ mesh and $6 \mathrm{~dB}$ for the worst case $45^{\circ}$ mesh (from the previous Section, only $\theta>55^{\circ}$ gives reasonably accurate results for this type of mesh). It should also be noted that these maximum differences occur at very small cell sizes, which in any case give a reflection less than $-40 \mathrm{~dB}$. At grid resolutions around ten cells/ wavelength the differences are minimal.

The results for the vertically-skewed mesh in Fig. 10 show higher reflections from the skewed regions (e.g. $-25 \mathrm{~dB}$ for the $60^{\circ}$ mesh with ten cells/wavelength), and indicate that skewing the mesh across a microstrip line in this fashion should be avoided.

\section{Conclusions}

This contribution has presented a detailed consideration of the dispersion in a non-orthogonal FDTD analysis of a microstrip line. Rather than employing a theoretical NDR, as in previous contributions (which limits the analysis to very simple meshes), dispersion was measured directly from the algorithm.

Dispersion in a mesh with a single skew angle compared well with the available closed-form NDR. Further analyses were carried out with multiple skew angles, and it has been observed that in order to keep the errors in the numerical phase velocity under a reasonable limit with a grid resolution of ten cells/wavelength, the mesh should be designed with cell angles $\theta$ in the range $55^{\circ}<\theta \leq 90^{\circ}$ for meshes skewed in a single plane, and $70^{\circ}<\theta \leq 90^{\circ}$ for meshes skewed in several planes.

Analyses with a non-uniformly skewed mesh confirm that the local dispersion is mainly governed by the worst skew angle in that region. Hence, the ranges above may be employed to guide the generation of each part of a more complex mesh.

Analyses without the interpolation step in the nonorthogonal algorithm showed a significant increase in numerical dispersion. This demonstrates that while the 
interpolation step is responsible for a large amount of the computational overheads in the non-orthogonal FDTD algorithm, it plays an important part in controlling numerical dispersion.

It is interesting to note that the closed-form NDR (which assumes plane wave propagation) does not give an entirely accurate estimate of the dispersion encountered in the nonorthogonal model of the microstrip line-this is evident in the differences between the two sets of curves in Fig. 3. It is believed that this small difference is because at metal edges and surfaces (such as in the microstrip example presented herein), the interpolation step in the non-orthogonal algorithm introduces a number of small additional errors. This phenomenon results in slightly increased dispersion compared to the plane wave case and is being investigated by the authors at the time of writing.

Furthermore, reflections from the interface between two skew regions have been estimated. Reflections from such interfaces were compared with results from an orthogonal mesh in order to estimate the effects of different cell sizes. It has been observed that the reflections from a horizontally skewed non-orthogonal mesh are slightly higher than for an orthogonal mesh with the same cell sizes; however, the increase did not exceed $2 \mathrm{~dB}$ for the transition from a $90^{\circ}$ mesh to a $60^{\circ}$ skewed mesh. With a vertically skewed mesh there is a significant increase in reflection, even for an angle of $60^{\circ}$.

\section{Acknowledgments}

The authors would like to thank Prof. McGeehan and Prof. White for provision of facilities at the University of Bristol, and EPSRC for supporting this work.

\section{References}

1 YEE, K.S.: 'Numerical solutions of initial boundary value problems involving Maxwell's equations in isotropic media', IEEE Trans. Antennas Propag., 1966, 14, pp. 302-307

2 JURGENS, T.G., TAFLOVE, A., UMASHANKAR, K.R., and MOORE, T.G.: 'Finite difference time domain modelling of curved surfaces', IEEE Trans. Antennas Propag., 1992, 40, pp. 357-366

$3 \mathrm{HAO}, \mathrm{Y}$., and RAILTON, C.J.: 'Analysing electromagnetic structures with curved boundaries on Cartesian FDTD meshes', IEEE Trans. Microw. Theory Tech., 1998, 46, pp. 82-88

4 GEDNEY, S., LANSING, F.S., and RASCOE, D.L.: 'Full wave analysis of microwave monolithic circuit devices using a generalised Yee algorithm based on an unstructured grid', IEEE Trans. Microw. Theory Tech., 1996, 44, pp. 1393-1400

5 RAY, S.L.: 'Numerical dispersion and stability characteristics of timedomain methods on non-orthogonal meshes', IEEE Trans. Antennas Propag 1993, 41, pp. 233-235

6 XIAO, F., and YABE, H.: 'Numerical dispersion relation for FDTD method in general curvilinear co-ordinates', IEEE Microw. Guid. Wave Lett., 1997, 7, pp. 48-50

7 SHI, H., and DREWNIAK, J.L.: 'Dispersion comparison for DSIand tensor-based non-orthogonal FDTD', IEEE Microw. Guid. Wave Lett., 1996, 6, pp. 193-195

8 TAFLOVE A. 'Review of the formulation and applications of the finite difference time-domain method for numerical modelling of electromagnetic wave interactions with arbitrary structures', Wave electromagnetic wave interactions
Motion, 1988,10, pp. $547-582$

9 SCHUHMANN, R., and WEILAND, T.: 'Stability of the FDTD algorithm on non-orthogonal grids related to the spatial interpolation scheme', IEEE Trans. Magn., 1998, 34, pp. 2751-2754

10 ZHANG, X, FANG, J MEI, K K and LIU, Y.: 'Calculations of the dispersive characteristics of microstrips by the time-domain finite difference method', IEEE Trans. Microw. Theory Tech., 1988, 36, pp. 263-267

11 NAVARRO, E.A., WU, C., CHUNG, P.Y., and LITVA, J.: 'Some considerations about the finite difference time domain method in general curvilinear co-ordinates', IEEE Microw. Guid. Wave Lett., 1994, 4, pp. 396-398 